\title{
La poesía infantil: a propósito de un libro de Concepción Ortega Casado
}

\section{Ghildren's poetry: about a book by Concepción Ortega Casado}

Miguel Gruz Giráldez

Universidad de Sevilla (España)

mcruz@us.e

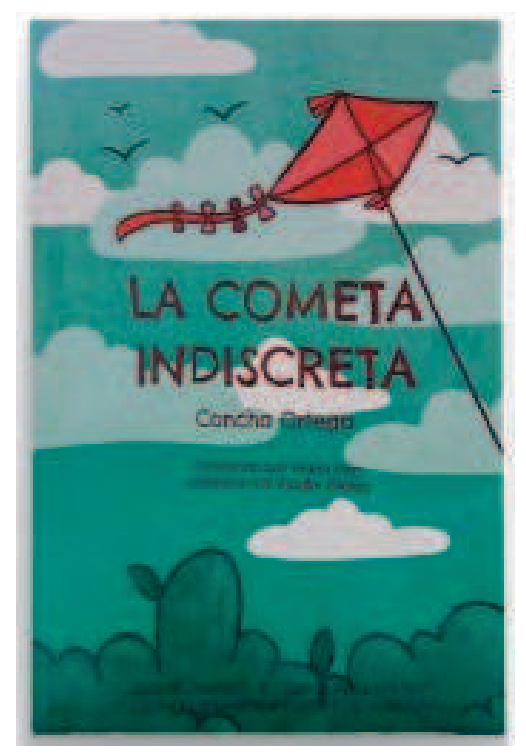

Reseña de la obra/ Book Review: Ortega Casado, Concepción (2020). La cometa indiscreta. Sevilla: En huida, 78 págs.

Concha Ortega Casado es una gran artista integral (pintora y poeta), que nos ha dado sobradas muestras a lo largo de los años de su capacidad creadora y de su buen hacer. Numerosas exposiciones en diversas y muy relevantes galerías certifican su condición de excepcional autora de óleos, acuarelas, acrílicos, que suponen un tratamiento difuminado del color y una figuración transida de poesía que abarca los bodegones, los retratos, las marinas o los paisajes. Su obra se caracteriza por las pinceladas fluidas, con escalas de colores matizadas de forma natural, y por la remisión a las más recónditas e invisibles relaciones entre las cosas del mundo, entendidas estas siempre como fuerzas y poderes elementales, que nos permiten captar el sentido simbólico de la realidad entrelazada con el arte, y lo que nos une de un modo indefectible a ellas: la emoción. Sus cuadros cuelgan hoy en importantes sedes de organismos oficiales y en las colecciones privadas de expertos y amantes de su pintura, tanto en España como en el extranjero.

Tal vez esta misma sensibilidad plástica la llevó a explorar también otros senderos del arte, como la literatura y -más en concreto- la poesía. Durante mucho tiempo fue una actividad cultivada por ella en silencio, hasta que hace poco empezó a darla a conocer. Desde entonces, Concha Ortega tiene poemas publicados en obras colectivas y en revistas, así como en blogs de Internet, y dos libros ya en solitario: El lugar de las dudas (2016) y La ausencia que me habita (2019), que han recibido 
una excelente acogida por parte del público y de la crítica especializada. En ellos muestra un dominio extraordinario de la forma (es una magnífica sonetista -una de las mejores de la poesía actual- en todas sus modalidades, aunque también cultiva con gran soltura y sentido rítmico el verso libre), y sus temas preferidos son los derivados de la indagación en su rico mundo interior: el tiempo ya ido, los recuerdos, el amor, la naturaleza, el sentido de la vida...

$\mathrm{Y}$ ahora nos sorprende con un nuevo poemario que de alguna manera supone un giro - pero no una ruptura- en su trayectoria, pues La cometa indiscreta es un libro de poesía infantil. La autora, madre y abuela, ama a los niños, con los que ha tenido siempre un trato continuado, y por eso mismo su incursión en este universo del imaginario infantil viene a ser una profundización en una de las líneas temáticas de su poesía: el afecto a los seres queridos.

Pero antes de seguir, conviene que nos detengamos en reflexionar sobre la literatura infantil. El concepto de literatura es muy amplio y complejo, ya que, antiguamente, se consideraba literatura, palabra derivada del latín littera, cuyo significado es 'letra', a todo tipo de escritura, sin distinción de su contenido. Actualmente, conocemos bien que la literatura también existe en el ámbito oral, por lo que la anterior concepción se ve ampliada. Por lo tanto, podemos definir la literatura como la serie de las obras de imaginación, de ficción, compuestas con voluntad de estilo, es decir, con la voluntad artística de crear belleza. Con esta definición más precisa se elimina cualquier tipo de comunicación (oral o escrita) de uso coloquial en el día a día, puesto que el fin esencial de la literatura es la belleza.

Pero si hablamos de literatura infantil es necesario también delimitar este concepto, pues resulta demasiado ambiguo. Porque el término literatura infantil comprende por lo menos tres clases de obras: una son los textos escritos por niños y adolescentes; otra los elaborados por adultos para el público infantil, como un tipo especial de lector modelo (según nos explican Umberto Eco y la estética de la recepción); y la tercera incluye los textos que la tradición literaria ha considerado como adecuados para los más pequeños. Y en el segundo sentido, sobre todo cuando se requiere la precisión, es frecuente hablar también de literatura para niños.

Ahora bien, la literatura para niños no es algo ciertamente menor, ni tampoco fácil: al contrario, es algo muy serio y de mucha trascendencia, pues va dirigida a quienes más queremos y a los que tratamos de formar a partir de una visión del mundo adaptada a su sensibilidad y a su mentalidad en proceso de configuración. La literatura infantil puede definirse como una actividad creadora que se dirige a los niños, que se compone de toda una serie de elementos como el lenguaje (verbal no verbal) o la imagen gráfica para transmitir sentimientos y valores, y constituye una muy eficaz herramienta educativa, puesto que conecta al niño con el mundo del arte, le dota de sensibilidad estética, provoca su reflexión, potencia también su capacidad para emocionarse, reír o llorar, comprender diferentes visiones y realidades de la vida y del mundo, lo introduce en la cultura a través de historias de la vida y el universo, 
tanto del entorno más próximo que le rodea como de otras tradiciones y otros pueblos. Por tanto, fíjense si es algo serio y difícil escribir un libro para los niños.

Encontramos que la literatura está presente en nuestra vida desde los primeros años a través de la poesía y el cuento. Este se clasifica dentro de los textos narrativos, y es, junto a la novela, una de las principales formas narrativas actuales, generalmente escritas en prosa. Se trata de un relato breve donde se presenta una trama muy condensada con un vocabulario accesible a niños y niñas.

Y si hablamos de poesía infantil, el reto es aún mayor, pues a través de la rima y la sonoridad hay que captar la atención de esos pequeños lectores y oyentes que buscan en el poema gratificarse y satisfacer su curiosidad. Leer o recitar una poesía representa un esfuerzo muy positivo para los niños, supone memorizar los versos y después declamarlos haciendo uso de la expresividad corporal. Además les ayuda, como hemos dicho, a expresar sus sentimientos y comprender mejor las pasiones y emociones.

La poesía para niños puede ser uno de los mejores medios para fomentar el gusto por la lectura entre los pequeños. Es difícil incentivar a un niño a leer algo que no le atrae, mientras que con la poesía los niños quedan encantados con la belleza y la armonía que transmite, además de la brevedad de sus textos. Esto es un reto siempre, pero más hoy, en un mundo en el que los pequeños están fascinados con las nuevas tecnologías.

Nada hay más difícil que lograr atraer (y sobre todo mantener) la atención de los pequeños hacia una actividad. Y mucho más si ésta se fundamenta en la palabra, para muchos de ellos todavía hablada, pues, no han aprendido aún a leer por sí mismos. De ahí que un libro de poesía infantil suponga un claro reto para el escritor que aborda un empeño tan exigente.

Sostiene Freire que la educación es un acto de amor, por lo tanto un acto de valor. El autor de un libro infantil no solo ha de tener un conocimiento amplio de la literatura y sus técnicas expresivas, sino un amor inmenso a los niños, a cuya educación quiere contribuir a través de su obra, y un buen bagaje de ternura y paciencia, fruto de un trato continuo con los pequeños, que le permite comprender su mentalidad y ponerse al nivel de sus gustos y exigencias. La autora (artista completa) es madre y también abuela, y esta proximidad y la cercanía a los niños más queridos a lo largo de toda su vida la ha hecho explorar este difícil camino.

La poesía infantil ha sido escrita para ser leída, comprendida y meditada. Es un verdadero placer el poder adentrarse en el mundo de los versos, de las rimas y de la expresión del arte literario dirigido de forma específica a los niños. Solo así se puede disfrutar verdaderamente del sentido y del juego de sus palabras y del lenguaje. La poesía abre las puertas a los niños a un mundo de versos y rimas, donde priman la fantasía y la expresión, y donde los juegos verbales y el lenguaje son los verdaderos protagonistas. 
extensos capítulos un análisis certero y riguroso de la intervención socioeducativa, de su planificación y, más exhaustivamente, de su evaluación.

La obra, coordinada por las profesoras Martín Cuadrado y Rubio Roldán, es el resultado del encomiable esfuerzo de un grupo de profesoras y profesores de la UNED por ofrecer a los estudiantes un texto que les proporcione sólidas bases conceptuales, a la vez que recursos prácticos y materiales útiles para una práctica profesional que responda a las necesidades y demandas actuales de los diferentes colectivos sociales y contextos de intervención socioeducativa en un escenario social, económico y cultural cambiante, fluido e incierto.

En este sentido, se puede considerar como un gran acierto la organización del material en dos volúmenes. Un primer volumen, que es el objeto de esta reseña, de carácter teórico y conceptual en el que se abordan las principales cuestiones que los futuros educadores y educadoras sociales han de conocer para diseñar, ejecutar y evaluar una intervención socioeducativa de calidad.

El segundo volumen tiene un marcado carácter práctico y aplicado y es, por tanto, el complemento justo y necesario de los aspectos más teóricos y conceptuales abordados en el primero. El análisis y valoración de la práctica profesional de la educación social en diferentes contextos y ámbitos; así como el conocimiento de instrumentos, recursos y materiales adaptados a las particularidades de esos contextos.

El texto está concebido como un manual universitario específicamente pensado para los estudiantes de la UNED aunque, sin duda, podrá ser de gran utilidad también para estudiantes de otras universidades, educadores y educadoras sociales en activo y, en general, para cualquier profesional de la acción social. Cada tema se abre con un mapa conceptual que permite un acercamiento visual a los principales conceptos del tema y sus relaciones al que sigue la relación de los objetivos generales y específicos que el estudiante ha de conseguir. La introducción incluye también las palabras clave e ideas básicas del tema. El núcleo de cada tema lo constituye, lógicamente, el desarrollo de los contenidos. La identidad profesional del educador y educadora social; el concepto y los modelos de intervención socioeducativa; la planificación de la intervención socioeducativa en planes, programas, proyectos, ámbitos y agentes; el sentido, funciones y modalidades de la evaluación en la intervención socioeducativa, así como los procedimientos, técnicas e instrumentos para llevarla a cabo y, finalmente, la autoevaluación de agentes e instituciones son los núcleos temáticos que se abordan desde las aportaciones más recientes de distintas disciplinas y el conocimiento construido desde la rica y variada práctica profesional de la educación social en las últimas décadas.

Ofrecer unos contenidos significativos, relevantes y actualizados es un requisito necesario, pero no suficiente, para facilitar el aprendizaje autónomo y autorregulado. Por este motivo, resultan muy pertinentes los elementos que tratan de facilitar el estudio a distancia: las lectura recomendadas para profundizar en las cuestiones tratadas a lo largo del tema (si el estudiante tiene tiempo e interés); las actividades 
de activación de los conocimientos previos, así como las de afianzamiento y consolidación de lo aprendido; los ejercicios de autoevaluación; el resumen en el que se recoge lo más significativo de los aspectos tratados; el glosario que incorpora los términos más importantes del tema y, finalmente, el solucionario de los ejercicios de autoevaluación. Todo ello está concebido con gran acierto para facilitar que el estudiante asimile, por un lado, los aspectos conceptuales y teóricos del tema y, por otro, compruebe por sí mismo su progreso en el estudio y aprendizaje.

El manual trata de responder a una serie de interrogantes que todo educador social ha de plantearse: ¿Quién soy como profesional de la educación social?, ¿qué funciones y competencias tendré que desarrollar en el ejercicio de mi profesión?, ¿cuáles son los elementos que definen mi actividad profesional?, ¿en qué modelos puedo sustentar mi práctica?, ¿cómo puedo planificar mis actuaciones?, ¿qué elementos tengo que tener en cuenta para planificar, aplicar y evaluar un proyecto de intervención socioeducativa?, ¿cuál es el sentido de la evaluación en la educación social y cómo puedo llevarla a cabo?, ¿qué procedimientos, técnicas e instrumentos puedo utilizar para ello?, ¿cómo puede la autoevaluación ayudarme a mejorar mi desempeño profesional?. El lector podrá encontrar en el texto cumplida respuesta a estas cuestiones y otras conexas.

En el primer capítulo, las profesoras Martín Cuadrado y García Vargas exponen, desde un enfoque evolutivo y multidimensional, la identidad profesional del educador social como agente de la acción socioeducativa, sus funciones y competencias, las variables que intervienen en la construcción de la identidad profesional y, en particular, el papel de las prácticas externas como un primer acercamiento del estudiante a la realidad de la profesión e invitan a seguir profundizando e investigando tanto desde la práctica de la profesión como desde la reflexión académica en el papel que los educadores sociales deben desempeñar en la construcción de una sociedad más justa y más igualitaria.

También con un carácter introductorio en el conjunto del manual, Martín Cuadrado y Rubio Roldán exponen, en el capítulo 2, una amplia visión de distintas aproximaciones al concepto de intervención socioeducativa, de los elementos que la definen y de los modelos teóricos desde los que puede ser abordada.

En el capítulo 3, la profesora Pérez Sánchez, partiendo de una concepción amplia de educación social, inspirada en la definición de Gómez Serra (2003), según la cual la educación social se dirige tanto a cualquier persona en cualquier espacio social como a quienes presentan necesidades particulares en el proceso de integración social, ofrece un completo análisis de las áreas de intervención en educación social, a partir de las propuestas de diversos autores (Froufe, 1997; Gómez Serra, 2003; Pérez Serrano, 2003; Ventosa, 2011), que da como resultado la agrupación en educación social especializada, educación de personas adultas, animación sociocultural y formación laboral los ámbitos de intervención socioeducativa. Asimismo, presenta una oportuna y necesaria clarificación de los términos que se utilizan habitualmente 
en lo relativo a la planificación de las intervenciones socioeducativas y aporta distintas definiciones del concepto de planificación, los principios que se deben tener en cuenta para acometerla, así como las fases y etapas que es necesario conocer para elaborar proyectos de intervención socioeducativa, aspecto en el que destaca por su claridad y utilidad la propuesta de Pérez Serrano (2016).

El capítulo dedicado a la evaluación se inicia con una afirmación recogida por el profesor Cardona que pone de manifiesto la necesidad de construir conocimiento específico sobre la evaluación en la educación social: "en educación social la cultura de la evaluación se enfrenta al desafio de ir construyendo su desarrollo" (Gomez, 2005-06, Martín, 2016). Y es que si bien contamos con un amplio conocimiento teórico y de aplicación práctica sobre la evaluación en el ámbito de la educación formal dentro del sistema educativo (evaluación de los aprendizajes, evaluación de centros, evaluación del profesorado, etc.), en el ámbito de la educación social se han de adoptar los paradigmas y modelos de evaluación que mejor respondan a las particularidades de los ámbitos y contextos y, así mismo, se han de adaptar los procedimientos, técnicas e instrumentos para llevarla a cabo.

Continuando con la evaluación, pero a un nivel de mayor concreción, el profesor Cabrerizo sistematiza, en el capítulo 5, los procedimientos, técnicas e instrumentos más utilizados en la evaluación de la intervención socioeducativa que todo profesional de la educación social debe conocer para utilizar en cada caso aquel que mejor se ajuste al contexto y situación a evaluar. Parte de la delimitación conceptual de los términos procedimiento, técnica e instrumento y ofrece distintas clasificaciones de técnicas e instrumentos para la evaluación de agentes, ámbitos y proyectos, a la vez que facilita criterios y variables a tener en cuenta para la selección del más adecuado en cada caso. La segunda parte del capítulo está dedicada a la exposición de la observación y la interrogación como procedimientos de evaluación y, dentro de cada uno de ellos, se analizan las técnicas, instrumentos y criterios para una aplicación correcta.

El mismo profesor Cabrerizo analiza, en el capítulo 6, la autoevaluación de agentes y de instituciones como un factor de calidad de la intervención socioeducativa. Sin duda alguna, la evaluación de la intervención socioeducativa sería incompleta si las instituciones o administraciones que promueven proyectos no rindieran cuentas a la sociedad a través de la evaluación de su idoneidad y calidad y si el agente no autoevalúa su actuación como profesional, pues la autoevaluación es garantía de la mejora continua en habilidades, competencias y actitudes.

En su conjunto, la obra constituye una valioso material de estudio; ofrece, desde luego, un conocimiento amplio, riguroso y actualizado en todos y cada uno de los temas que aborda pero, me atrevería a decir que destaca, sobre todo, por la pasión y el compromiso por la educación social del presente y del futuro de todas las personas que han participado en su elaboración, que es tanto como decir compromiso por la construcción de una sociedad inclusiva, solidaria y justa a través de la formación de los profesionales dedicados a la intervención socioeducativa. 